\title{
On sequential parameter estimation for some linear stochastic differential equations with time delay *
}

\author{
Uwe Küchler* \\ Humboldt University, Berlin \\ Institute of Mathematics
}

\author{
Vjatscheslav A. Vasil'iev** \\ Tomsk State University, \\ Dept. of Applied Mathematics \\ and Cybernetics
}

November 3, 1998

\begin{abstract}
We consider the parameter estimation problem for the scalar diffusion type process described by the stochastic equation with time delay

$$
d X(t)=\sum_{i=0}^{m} \vartheta_{i} X\left(t-r_{i}\right) d t+d W(t)
$$

The asymptotic behavior of the classical maximum likelihood estimator (MLE) very depends on the true values of parameter $\vartheta=\left(\vartheta_{0}, \vartheta_{1}, \ldots, \vartheta_{m}\right)^{\prime}$.

Here we construct a sequential MLE with preassigned least square accuracy for the so-called stationary and the periodic cases of the solution $X(\cdot)$. The limit behaviour of the duration of the procedure with given accuracy is obtained.
\end{abstract}

Keywords: stochastic differential equations; time delay; maximum likelihood estimator; sequential analysis; least square accuracy.

\footnotetext{
* This work was supported by the Deutsche Forschungsgemeinschaft, Sonderforschungsbereich 373

"Quantifikation und Simulation ökonomischer Prozesse", Berlin, Germany
} 


\section{Introduction}

Assume $\left(W(t), \mathcal{F}_{t}, t \geq 0\right)$ is a realvalued Wiener process on a filtered probability space $\left(\Omega, \mathcal{F},\left(\mathcal{F}_{t}, t \geq 0\right), P\right)$ and $(X(t), t \geq-r)$ satisfies the following differential equation with time delay

* Unter den Linden 6, D-10099 Berlin, Germany

** Lenina 36, 634050 Tomsk, Russia.

$$
\left.\begin{array}{l}
d X(t)=\sum_{i=0}^{m} \vartheta_{i} X\left(t-r_{i}\right) d t+d W(t), t \geq 0, \\
X(s)=X_{0}(s), s \in[-r, 0] .
\end{array}\right\}
$$

The parameters $r_{i}, \vartheta_{i}, i=0, \ldots, m$ are real numbers with $0=r_{0}<r_{1}<\ldots<$ $r_{m}=: r$, if $m \geq 1$ and $r_{0}=r=0$ if $m=0$. The initial process $\left(X_{0}(s), s \in[-r, 0]\right)$ is supposed to be cadlag and all $X_{0}(s), s \in[-r, 0]$ are assumed to be $\mathcal{F}_{0}$-measurable. Moreover assume that

$$
E \int_{-1}^{0} X_{0}^{2}(s) d s<\infty
$$

The equation (1) is a special case of so-called affine stochastic differential equation studied in detail e.g. in $[\mathrm{Mo} / \mathrm{Sch}]$ and $[\mathrm{Mo}]$. In particular it holds, that (1) has a uniquely determined solution $(X(t), t \geq-r)$ having the representation

$$
\left.\begin{array}{rlr}
X(t)= & \sum_{j=0}^{m} \vartheta_{j} \int_{-r_{j}}^{0} x_{0}\left(t-s-r_{j}\right) X_{0}(s) d s+ & \\
& +x_{0}(t) X_{0}(0)+\int_{0}^{t} x_{0}(t-s) d W(s), & t>0 \\
X(t)= & X_{0}(t), & t \in[-r, 0]
\end{array}\right\}
$$

and satisfying $E \int_{0}^{T} X^{2}(s) d s<\infty$ for every $T$ with $0<T<\infty$. Here the function $x_{0}(\cdot)$ denotes the fundamental solution of the corresponding to (1) linear deterministic equation

$$
\begin{aligned}
& x_{0}(t)=1+\sum_{j=0}^{m} \int_{0}^{t} \vartheta_{j} x_{0}\left(t-r_{j}\right), \quad t \geq 0, \\
& x_{0}(s)=0, s \in[-r, 0) ; x_{0}(0)=1 .
\end{aligned}
$$

(see $[\mathrm{Ha} / \mathrm{Ve}]$ for details on $(3)$ ).

Fix a subject $\Theta$ of $R^{m+1}$ and assume the vector $\vartheta=\left(\vartheta_{0}, \vartheta_{1}, \ldots, \vartheta_{m}\right)^{\prime} \in \Theta$ is unknown and has to be estimated based on the observation $(X(t))$. The delay times $r_{i}$ are supposed to be known.

The measures $P_{\vartheta}, \vartheta \in R^{m+1}$ generated by the solutions of (1) form an exponential family in the sense of [Ku/So]. Thus, one possibility to estimate $\vartheta$ is to use the maximum likelihood method. The corresponding log-likelihood-function is given by

$$
\ell_{t}(\vartheta)=\vartheta^{\prime} \Phi(t)-\frac{1}{2} \vartheta^{\prime} G(t) \vartheta \quad, \quad \vartheta \in \Theta, t>0,
$$


where

$$
\Phi(t)=\left(\int_{0}^{t} X\left(s-r_{i}\right) d X(s), i=0, \ldots, m\right)^{\prime}
$$

and

$$
G(t)=\left(\int_{0}^{t} X\left(s-r_{i}\right) X\left(s-r_{j}\right) d s, i, j=0, \ldots, m\right)
$$

denotes the Fisher information matrix (for details see $[\mathrm{Gu} / \mathrm{Ku}]$ and $[\mathrm{Ku} / \mathrm{So}]$ ). Another method is provided by sequential estimation. Sequential estimation of onedimensional parameters in exponential families of processes have been studied e.g. in [Li/Sh] and [Nov], see also [Ku/So] (1997), Chapter 10. The more-dimensional parameter case cannot be treated in the same way. Indeed, the construction of the stopping time for the observation in these papers very uses the one-dimensionality of the Fisher information. For processes arising from linear stochastic differential equations without time delay having more-dimensional parameters, sequential methods have been developed in [Ko/Pe] (1985), (1987), (1992).

Here we shall extend these results to equations of the type (1). We shall construct for every $\varepsilon>0$ a sequential procedure $\vartheta^{*}$ to estimate $\vartheta$ with $\varepsilon$-accuracy in the square mean sense, i.e. with $E\left[\vartheta^{*}-\vartheta\right]^{2} \leq \varepsilon$.

The method used below is a two step construction of a random time, where the first step uses the trace of the Fisher information matrix and follows the line of the one-dimensional case mentioned above.

A generalization of the sequential estimators, constructed in the sequel, to differential equations of the type (1) but based on noisy observations, will be presented in a subsequent paper.

\section{Results}

Consider the process $(X(t), t \geq-r)$ described by equation (1) above.

Throughout this paper we suppose that the following assumption holds.

Assumption (A) : For every $\vartheta \in \Theta$ there exist a (deterministic scalar) positive increasing function $\varphi(\cdot)$ on $[0, \infty)$ with $\lim _{T \rightarrow \infty} \varphi(T)=\infty$ and a possibly random $(m+1) \times(m+1)$-matrix function $I_{\infty}(T), T \in[0, \infty)$, being continuous periodic with period $\Delta \geq 0\left(\Delta=0\right.$ means $I_{\infty}(T) \equiv I_{\infty}(0)$ and positive definite for every $T$. Moreover, it holds

$$
\lim _{T \rightarrow \infty}\left|\frac{G(T)}{\varphi(T)}-I_{\infty}(T)\right|=0 \quad \text { a.s. }
$$

The assumption $(A)$ is satisfied under further restrictions on $\Theta$ only. For example, if $m=1$ then it holds exactly in the following two cases.

Consider the set $\Lambda$ of all complex roots of the socalled characteristic equation

$$
\lambda-\vartheta_{0}-\vartheta_{1} \mathrm{e}^{-\lambda r}=0
$$


and put $v_{0}=v_{0}(\vartheta)=\max \{\operatorname{Re} \lambda \mid \lambda \in \Lambda\}$. It can be easily shown that $v_{0}<\infty$. Then (A) holds for $\Theta=\left\{\vartheta \in R^{2} \mid v_{0}(\vartheta)<0\right.$ or $\left[v_{0}(\vartheta)>0\right.$ and $\left.\left.v_{0}(\vartheta) \notin \Lambda\right]\right\}$, see $[\mathrm{Gu} / \mathrm{Ku}]$ (1998) for details. If $v_{0}<0$ then the equation (1) admits a stationary solution and every solution tends to it in distribution, moreover we have $\Delta=0$, we call this case the "stationary case". If $v_{0}>0$ and $v_{0} \notin \Lambda$ the equality (5) is valid with some $\Delta>0$. We denote this case as the "periodic" one.

A similar picture appears in the classical moredimensional linear equation

$$
d X(t)=A X(t) d t+d W(t), t \geq 0, \quad X(0)=X_{0}
$$

with the Fisher information matrix

$$
\Gamma(T)=\int_{0}^{T} X(T) X^{\prime}(T) d t .
$$

Here $W(\cdot)$ is a $d$-dimensional standard Wiener process and $A$ a given $d \times d$ matrix. Let $\lambda_{\max }$ and $\lambda_{\min }$ be eigenvalues of $A$ having the maximal and minimal absolute value under all of eigenvalues of $e^{A}$, respectively. It is well known that the limiting matrix $\lim _{T \rightarrow \infty} T^{-1} \Gamma(T)$ exist and is a positive definite deterministic matrix in the stable case $\left(\operatorname{Re} \lambda_{\max }<0\right)$ and $\Gamma(T)$ increase exponentially in the unstable case $\left(\operatorname{Re} \lambda_{\min }>0\right)$. Note that for stable case the sequential parameter estimation problem of matrix $A$ was considered in $[\mathrm{Ko} / \mathrm{Pe}]$ (1985), for the scalar model in [Nov] and [Li/Sh], for unstable case in [Ko/Pe] (1987) and in mixed case $\left(\operatorname{Re} \lambda_{\max }>0, \operatorname{Re} \lambda_{\min }<0\right.$ and $\lambda+\mu \neq 0$ for all eigenvalues $\lambda, \mu$ of $\left.A\right)$ in $[\mathrm{Ko} / \mathrm{Pe}]$ (1992).

The sequential estimation problem for the matrix $A$ in the stable case by noisy observations was studed in [Va/Ko] (1987) and [Va/Ko] (1990).

Let us return to the study of (1) and let Assumption (A) be true.

To estimate $\vartheta$ with pressigned accuracy $\varepsilon>0$ we shall start with the maximum likelihood estimator of $\vartheta$ for the given lenght $T$ of observation defined by the equality

$$
\hat{\vartheta}(T)=G^{-1}(T) \Phi(T), T>0 .
$$

From (1) and (6) we find the deviation of the estimator $\hat{\vartheta}(T)$ from $\vartheta$ :

$$
\hat{\vartheta}(T)-\vartheta=G^{-1}(T) \zeta(T)
$$

with

$$
\zeta(T)=\int_{0}^{T} Z(t) d W(t), Z(t)=\left(X(t), X\left(t-r_{1}\right), \ldots, X\left(t-r_{m}\right)\right)^{\prime} .
$$

Now we make a time substitution which enables us to control the second moments of the noise $\zeta$.

Fix an arbitrary increasing sequence $\left(c_{n}\right)_{n \geq 1}$ of reals tending to infinity. Let us define the sequence of $\left(\mathcal{F}_{t}\right)$-stopping times $\left(\tau_{\varepsilon}(n), n \geq 1\right)$ as follows

$$
\tau_{\varepsilon}(n)=\inf \left\{T>0: \operatorname{tr} G(T)=\varepsilon^{-1} c_{n}\right\},
$$


These moments are finite a.s. due to the condition (5).

One can easily verify that for any $\varepsilon>0$ the sequence $\left(\zeta\left(\tau_{\varepsilon}(n)\right), n \geq 1\right)$ satisfies the equalities

$$
E_{\vartheta}\left\|\zeta\left(\tau_{\varepsilon}(n)\right)\right\|^{2}=\varepsilon^{-1} c_{n}, n \geq 1 .
$$

(Throughout this paper $\|\cdot\|$ denotes the Euclidian norm.)

The equalities (9) suggest that the estimation of the parameter $\vartheta$ should be performed at the moments $\tau_{\varepsilon}(n)$ :

$$
\vartheta_{n}(\varepsilon)=\hat{\vartheta}\left(\tau_{\varepsilon}(n)\right), n \geq 1 .
$$

According to (7) in order to obtain the estimates with fixed least square deviation now one should control the behaviour of the sequence of random matrices $\left(G^{-1}\left(\tau_{\varepsilon}(n)\right), n \geq 1\right)$. It can be achieved by conducting the observations up to the moment $\tau_{\varepsilon}(n)$ with a specially choosen number $n$. Let

$$
\nu_{\varepsilon}=\inf \left\{N \geq 1: S_{N}(\varepsilon) \geq \varrho\right\},
$$

where $S_{N}(\varepsilon)=\sum_{n=1}^{N} \beta_{n}^{2}(\varepsilon), \beta_{n}^{2}(\varepsilon)=\left(\varepsilon \cdot c_{n}^{-1}\right)^{2} .\left\|G^{-1}\left(\tau_{\varepsilon}(n)\right)\right\|^{-2}, \varrho=\sum_{n \geq 1} 1 / c_{n}$.

The sequential plan $\left(T(\varepsilon), \vartheta_{\varepsilon}^{*}\right)$ of estimation of the vector $\vartheta$ will be defined by

$$
T(\varepsilon)=\tau_{\varepsilon}\left(\nu_{\varepsilon}\right), \vartheta_{\varepsilon}^{*}=S_{\nu_{\varepsilon}}^{-1} \sum_{n=1}^{\nu_{\varepsilon}} \beta_{n}^{2}(\varepsilon) \cdot \vartheta_{n}(\varepsilon)
$$

Obviously, $\nu_{\varepsilon}$ is a $\left(\mathcal{F}_{\tau_{n}(\varepsilon)}\right)$-stopping time, and therefore, by construction, $T(\varepsilon)$ turns out to be an $\left(\mathcal{F}_{t}\right)$-stopping time.

In such a way the sequential estimate $\vartheta_{\varepsilon}^{*}$ is a random weighted mean of the maximum likelihood estimates, calculated at the stopping times $\tau_{\varepsilon}(n), n \geq 1$.

The following theorem summarizes the main result.

Theorem 1. Assume that Assumption A holds. Then for any $\varepsilon>0$ and any $\vartheta \in \Theta$ the sequential estimation plan (1D) of $\vartheta$ possesses the properties:

$1^{\circ} . T(\varepsilon)<\infty \quad P_{\vartheta}-$ a.s.,

$2^{\circ} . E_{\vartheta}\left\|\vartheta_{\varepsilon}^{*}-\vartheta\right\|^{2} \leq \varepsilon$

and the following inequalities hold $P_{\vartheta}-$ a.s.

$3^{\circ} .0<\frac{\lim _{\varepsilon \rightarrow 0}}{\varepsilon} \cdot \varphi(T(\varepsilon)) \leq \varlimsup_{\varepsilon \rightarrow 0} \varepsilon \cdot \varphi(T(\varepsilon))<\infty$.

Proof. $1^{\circ}$. Let us verify the finiteness of $T(\varepsilon)=\tau_{\varepsilon}\left(\nu_{\varepsilon}\right)$. While the moments $\tau_{\varepsilon}(n)$ are finite for all $n \geq 1$, it suffices to establish the finiteness of the moment $\nu_{\varepsilon}$. Making 
use of the definition $(9)$ of $\tau_{\varepsilon}(n)$ and the condition (5) we have

$$
\lim _{n \rightarrow \infty}\left|\frac{\varepsilon^{-1} \cdot c_{n}}{\varphi\left(\tau_{\varepsilon}(n)\right)}-\operatorname{tr} I_{\infty}\left(\tau_{\varepsilon}(n)\right)\right|=0 \quad \text { a.s. }
$$

and as follows by the definition of $\beta_{n}^{2}(\varepsilon)$

$$
\lim _{n \rightarrow \infty}\left|\beta_{n}^{2}(\varepsilon)-\beta^{2}\left(\tau_{\varepsilon}(n)\right)\right|=0 \quad \text { a.s. }
$$

where

$$
\beta^{2}(u)=\left[\operatorname{tr} I_{\infty}(u) \cdot\left\|I_{\infty}^{-1}(u)\right\|\right]^{-2} .
$$

Note that by the conditions on the matrix function $I_{\infty}(u)$ we have

$$
\inf _{u \in R^{1}} \beta^{2}(u)>0 .
$$

Then $\sum_{n=1}^{\infty} \beta_{n}^{2}(\varepsilon)=\infty$ a.s. and for all $\varepsilon>0$ the moments $\nu_{\varepsilon}$ and $T(\varepsilon)$ are finite a.s.

$2^{\circ}$. Now we estimate the mean square deviation of $\vartheta_{\varepsilon}^{*}$. From (7), (9), (12) and by definitions of $\nu_{\varepsilon}, \beta_{n}$ and $\varrho$ it follows that

$$
\begin{aligned}
& E_{\vartheta}\left\|\vartheta_{\varepsilon}^{*}-\vartheta\right\|^{2}=E_{\vartheta} S_{\nu_{\varepsilon}}^{-2}\left\|\sum_{n=1}^{\nu_{\varepsilon}} \beta_{n}^{2}(\varepsilon)\left(\vartheta_{n}(\varepsilon)-\vartheta\right)\right\|^{2} \leq \\
& \leq E_{\vartheta} S_{\nu_{\varepsilon}}^{-1} \sum_{n \geq 1} \beta_{n}^{2}(\varepsilon)\left\|\vartheta_{n}(\varepsilon)-\vartheta\right\|^{2} \leq \varrho^{-1} \sum_{n \geq 1} E_{\vartheta} \beta_{n}^{2}(\varepsilon) \cdot \\
& \cdot\left\|G^{-1}\left(\tau_{\varepsilon}(n)\right)\right\|^{2} \cdot\left\|\zeta\left(\tau_{\varepsilon}(n)\right)\right\|^{2}=\varepsilon^{2} \varrho^{-1} \sum_{n \geq 1} \frac{1}{c_{n}^{2}} E_{\vartheta}\left\|\zeta\left(\tau_{\varepsilon}(n)\right)\right\|^{2}= \\
& =\varepsilon \varrho^{-1} \sum_{n \geq 1} \frac{1}{c_{n}}=\varepsilon .
\end{aligned}
$$

For the first inequality we used the Cauchy-Bunjakovsky inequality.

$3^{\circ}$. In order to establish the limiting relationships for $T(\varepsilon)$ we note that as in (14) for all $n \geq 1$ it holds

$$
\lim _{\varepsilon \rightarrow 0}\left|\beta_{n}^{2}(\varepsilon)-\beta^{2}\left(\tau_{\varepsilon}(n)\right)\right|=0 \quad \text { a.s. }
$$

According to (16) and by the definition of the moment $\nu_{\varepsilon}$ for small but positive $\varepsilon$ we have the inequalities

$$
\nu^{\prime} \leq \nu_{\varepsilon} \leq \nu^{\prime \prime} \quad \text { a.s. }
$$

with

$$
\begin{aligned}
\nu^{\prime} & =\inf \left\{N \geq 1: N>\varrho\left[\sup _{u \in[0, \Delta)} \beta^{2}(u)\right]^{-1}\right\}-1 \\
\nu^{\prime \prime} & =\inf \left\{N \geq 1: N>\varrho\left[\inf _{u \in[0, \Delta)} \beta^{2}(u)\right]^{-1}\right\} .
\end{aligned}
$$


Similar (13) we can obtain

$$
\lim _{\varepsilon \rightarrow 0}\left|\frac{\varepsilon^{-1} c_{\nu_{\varepsilon}}}{\varphi(T(\varepsilon))}-\operatorname{tr} I_{\infty}(T(\varepsilon))\right|=0 \quad \text { a.s. }
$$

From (17) and (18) follows the assertion $3^{\circ}$ of the Theorem 1

$$
0<\mu^{\prime} \leq \lim _{\varepsilon \rightarrow 0} \varepsilon \cdot \varphi(T(\varepsilon)) \leq \mu^{\prime \prime}<\infty,
$$

where

$$
\mu^{\prime}=c_{\nu^{\prime}} \cdot\left[\sup _{u \in[0, \Delta)} I_{\infty}(u)\right]^{-1}, \quad \mu^{\prime \prime}=c_{\nu^{\prime \prime}} \cdot\left[\inf _{u \in[0, \Delta)} I_{\infty}(u)\right]^{-1} .
$$

Theorem 1 is proved.

\section{Example}

Consider system (1) with $m=1, r_{1}=1$

$$
\left.\begin{array}{l}
d X(t)=\vartheta_{0} X(t) d t+\vartheta_{1} X(t-1) d t+d W(t), t \geq 0, \\
X(s)=X_{0}(s), s \in[-1,0] .
\end{array}\right\}
$$

Assume for reasons of of citation, that $X_{0}$ is continuous.

The sequential plan $\left(T(\varepsilon), \vartheta_{\varepsilon}^{*}\right)$ of estimation $\vartheta=\left(\vartheta_{0}, \vartheta_{1}\right)^{\prime}$ will be defined as (12) with the Fisher information matrix

$$
G(T)=\left(\begin{array}{ll}
\int_{0}^{T} X^{2}(t) d t & \int_{0}^{T} X(t) X(t-1) d t \\
\int_{0}^{T} X(t) X(t-1) d t & \int_{0}^{T} X^{2}(t-1) d t
\end{array}\right)
$$

$[\mathrm{Ku} / \mathrm{So}]$.

We can reformulated Theorem 1 for this cases as follows.

Theorem 2. Let the parameters $\vartheta_{0}$ and $\vartheta_{1}$ in (20) such that we have the stationary or periodic case (for the notation see chapter one). Then the sequential plan (12) of estimation $\vartheta=\left(\vartheta_{0}, \vartheta_{1}\right)^{\prime}$ possesses the properties:

$1^{\circ} . T(\varepsilon)<\infty \quad P_{\vartheta}-$ a.s.

$2^{\circ} \cdot E_{\vartheta}\left\|\vartheta_{\varepsilon}^{*}-\vartheta\right\|^{2} \leq \varepsilon$.

$3^{\circ}$. Besides the following limit inequalities

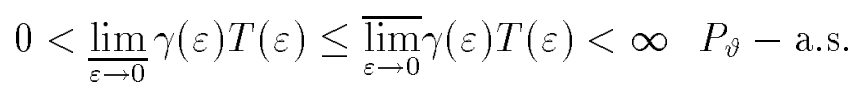


are fulfilled, where $\gamma(\varepsilon)=\varepsilon$ in the stationary case and $\gamma(\varepsilon)=\left(\ln \varepsilon^{-1}\right)^{-1}$ in the periodic case. Moreover, in the periodic case the limiting inequality

$$
\lim _{\varepsilon \rightarrow 0}\left|T(\varepsilon)-\frac{1}{2 v_{0}} \ln \varepsilon^{-1}\right|<\infty \quad \text { a.s. }
$$

holds.

Proof of $1^{\circ}-2^{\circ}$ : According to Theorem 1 the assertions $1^{\circ}$ and $2^{\circ}$ of Theorem 2 will be proved if the matrix $G(T)(21)$ satisfies the condition (5).

Now we establish the auxiliary equalities

$$
\lim _{T \rightarrow \infty} T^{-1} G(T)=I_{\infty} \quad \text { a.s. }
$$

for the stationary case and

$$
\lim _{T \rightarrow \infty}\left|\mathrm{e}^{-2 v_{0} T} G(T)-I_{\infty}(T)\right|=0 \quad \text { a.s. }
$$

for the periodic case, $v_{0}>0$.

Here

$$
I_{\infty}=\left(\begin{array}{ll}
\int_{0}^{\infty} x_{0}^{2}(t) d t & \int_{0}^{\infty} x_{0}(t) x_{0}(t+1) d t \\
\int_{0}^{\infty} x_{0}(t) x_{0}(t+1) d t & \int_{0}^{\infty} x_{0}^{2}(t) d t
\end{array}\right)
$$

and $I_{\infty}(T)$ is a periodic matrix

$$
\begin{gathered}
I_{\infty}(T)=\left(\begin{array}{cc}
g_{11}(T) & g_{12}(T) \\
g_{12}(T) & g_{22}(T)
\end{array}\right), \\
g_{i j}(T)=\int_{0}^{\infty} \mathrm{e}^{-2 v_{0} t} U_{i}(T-t) U_{j}(T-t) d t, i, j=0,2, \\
U_{i}(t)=\phi_{i}(t) X_{0}(0)+b \int_{-1}^{0} \phi_{i}(t-s-1) \mathrm{e}^{-v_{0}(s+1)} X_{0}(s) d s+\int_{0}^{\infty} \phi_{i}(t-s) \mathrm{e}^{-v_{0} s} d W(s), \\
\phi_{i}(t)=A_{i} \cdot \cos \left(\xi_{0} t\right)+B_{i} \cdot \sin \left(\xi_{0} t\right), \quad i=0,2, \\
A_{0}=\frac{2\left(v_{0}-a+1\right)}{\left(v_{0}-a+1\right)^{2}+\xi_{0}^{2}}, B_{0}=\frac{2 \xi_{0}}{\left(v_{0}-a+1\right)^{2}+\xi_{0}^{2}}, \\
\left(\begin{array}{l}
A_{2} \\
B_{2}
\end{array}\right)=\mathrm{e}^{-v_{0}\left(\begin{array}{cc}
\cos \xi_{0} & -\sin \xi_{0} \\
\sin \xi_{0} & \cos \xi_{0}
\end{array}\right)\left(\begin{array}{l}
A_{0} \\
B_{0}
\end{array}\right),}
\end{gathered}
$$




$$
\xi_{0}=\arg \left\{\operatorname{Im} \lambda \mid \lambda \in \Lambda, \operatorname{Re} \lambda=v_{0}, \operatorname{Im} \lambda>0\right\} .
$$

Taking into account the representation

$$
X(t)=x_{0}(t) X_{0}(0)+b \int_{-1}^{0} x_{0}(t-s-1) X_{0}(s) d s+\int_{0}^{t} x_{0}(t-s) d W(s)
$$

for the solution $(X(t), t \geq-1)$ of $(21)[\mathrm{Gu} / \mathrm{Ku}],[\mathrm{Ku} / \mathrm{So}]$ and the fact that in the stationary case

$$
\int_{0}^{\infty} x_{0}^{2}(t) d t<\infty
$$

we can see that

$$
\lim _{t \rightarrow \infty}|X(t)-Z(t)|=0 \quad \text { a.s. }
$$

where $Z(t)=\int_{-\infty}^{t} x_{0}(t-s) d W(s)$ is a stationary process with the correlation matrix $I_{\infty}$, which is ergodic [Gu/Ku], [Ku/So]. Then the equality (24) hold.

In the periodic case according to $[\mathrm{Gu} / \mathrm{Ku}]$

$$
x_{0}(t)=\phi_{0}(t) \mathrm{e}^{v_{0} t}+o\left(\mathrm{e}^{\gamma t}\right)
$$

and

$$
x_{0}(t-1)=\phi_{2}(t) \mathrm{e}^{v_{o} t}+o\left(\mathrm{e}^{\gamma t}\right)
$$

for some $\gamma$ with $\gamma<v_{0}$. Similar to Lemma 4.8 in [Gu/Ku] we can prove the equality

$$
\lim _{t \rightarrow \infty}\left|\mathrm{e}^{-\vartheta_{0} t} X(t)-U_{0}(t)\right|=0 \quad \text { a.s. }
$$

From here we have

$$
\begin{aligned}
& \lim _{t \rightarrow \infty}\left|\mathrm{e}^{-2 v_{0} T} \int_{0}^{T} X^{2}(t) d t-\int_{0}^{\infty} \mathrm{e}^{-2 v_{0} t} U^{2}(T-t) d t\right|= \\
& =\lim _{T \rightarrow \infty} \mid \int_{0}^{T} \mathrm{e}^{-2 v_{0}(T-t)}\left[\mathrm{e}^{-2 v_{0} t} X^{2}(t)-U^{2}(t)\right] d t+\int_{0}^{T} \mathrm{e}^{-2 v_{0}(T-t)} U^{2}(t) d t- \\
& -\int_{0}^{\infty} \mathrm{e}^{-2 v_{0} t} U^{2}(T-t) d t \mid=\lim _{T \rightarrow \infty} \int_{T}^{\infty} \mathrm{e}^{-2 v_{0} t} U^{2}(T-t) d t=0 \quad \text { a.s. }
\end{aligned}
$$

The other equations in (25) may be proved analogously. Note that according to $[\mathrm{Gu} / \mathrm{Ku}] I_{\infty}(u)>0$ for $u \in[0, \Delta)$ and the matrix function $I_{\infty}(u)$ is continuous on $R^{1}$. It follows $I_{\infty}(u)>0$ for $u \in[0, \Delta]$. Then (24), (25) and the conditions (5) for the matrix $G(T)$ defined by (21) are established. 
$3^{\circ}$. In order to obtain the exact limiting relationships for $T(\varepsilon)$ in the stationary case it suffices to note that by the definition of stopping times $\tau_{\varepsilon}(n)$ and (24) we get for all $n>1$

$$
\begin{array}{r}
\lim _{\varepsilon \rightarrow 0} \varepsilon \cdot \tau_{\varepsilon}(n)=c_{n} \cdot\left(\operatorname{tr} I_{\infty}\right)^{-1}>0 \text { a.s. } \\
\lim _{\varepsilon \rightarrow 0} \varepsilon \cdot G\left(\tau_{\varepsilon}(n)\right)=c_{n} \cdot\left(\operatorname{tr} I_{\infty}\right)^{-1} \cdot I_{\infty}>0 \text { a.s. }
\end{array}
$$

and as follows

$$
\lim _{\varepsilon \rightarrow 0} \beta_{n}^{2}(\varepsilon)=\left(\operatorname{tr} I_{\infty} \cdot\left\|I_{\infty}^{-1}\right\|\right)^{-2}>0 \quad \text { a.s. }
$$

Take into account that in this case $\varphi(T)=T$, from (8), (11), (24) and (28) we have

$$
\mu_{1} \leq \lim _{\varepsilon \rightarrow 0} \varepsilon \cdot T(\varepsilon) \leq \varlimsup_{\varepsilon \rightarrow 0} \varepsilon \cdot T(\varepsilon) \leq \mu_{2}
$$

with

$$
\begin{gathered}
\mu_{1}=c_{\nu-1} \cdot\left(\operatorname{tr} I_{\infty}\right)^{-1}, \mu_{2}=c_{\nu}\left(\operatorname{tr} I_{\infty}\right)^{-1}, \\
\nu=\inf \left\{N \geq 1: N>\varrho\left(\operatorname{tr} I_{\infty} \cdot\left\|I_{\infty}^{-1}\right\|\right)^{2}\right\} .
\end{gathered}
$$

Then the inequalities (22) for the stationary case hold.

Now we establish the assertion $3^{\circ}$ of Theorem 2 for the periodic case.

By the definition (8) and according to (25) we have

$$
\lim _{\varepsilon \rightarrow 0}\left|\varepsilon^{-1} c_{\nu_{\varepsilon}} \cdot \mathrm{e}^{-2 v_{0} T(\varepsilon)}-\operatorname{tr} I_{\infty}(T(\varepsilon))\right|=0 \quad \text { a.s. }
$$

Since $\inf _{u} \operatorname{tr} I_{\infty}(u)>0$ we can rewrite (31) in the form

$$
\lim _{\varepsilon \rightarrow 0}\left[T(\varepsilon)-\frac{1}{2 v_{0}} \ln \varepsilon^{-1}-\frac{1}{2 v_{0}} \ln c_{\nu_{\varepsilon}}+\frac{1}{2 v_{0}} \ln \operatorname{tr} I_{\infty}(T(\varepsilon))\right]=0 \quad \text { a.s. }
$$

From here and (17) we can obtain the relationships

$$
\lim _{\varepsilon \rightarrow 0}\left(\ln \varepsilon^{-1}\right)^{-1} \cdot T(\varepsilon)=\frac{1}{2 v_{0}} \quad \text { a.s. }
$$

and

$$
\tilde{\mu}_{1} \leq \lim _{\varepsilon \rightarrow 0}\left[T(\varepsilon)-\frac{1}{2 v_{0}} \ln \varepsilon^{-1}\right] \leq \varlimsup_{\varepsilon \rightarrow 0}\left[T(\varepsilon)-\frac{1}{2 v_{0}} \ln \varepsilon^{-1}\right] \leq \tilde{\mu}_{2}
$$

with

$$
\begin{aligned}
& \tilde{\mu}_{1}=\frac{1}{2 v_{0}} \ln c_{\nu^{\prime}}\left(\sup _{u \in[0, \Delta)} \operatorname{tr} I_{\infty}(u)\right)^{-1}, \\
& \tilde{\mu}_{2}=\frac{1}{2 v_{0}} \ln c_{\nu^{\prime \prime}}\left(\inf _{u \in[0, \Delta)} \operatorname{tr} I_{\infty}(u)\right)^{-1} .
\end{aligned}
$$


The assertion $3^{\circ}$ of Therem 2 is established. Theorem 2 is proved.

From Theorem 2 it follows that the duration $T(\varepsilon)$ of the sequential estimation has a nonrandom lower and upper bounds $\gamma^{-1}(\varepsilon) \tilde{\mu}_{1}$ and $\gamma^{-1}(\varepsilon) \tilde{\mu}_{2}$ respectively asymptotically. These bounds have the same increasing rate with $\varepsilon \rightarrow 0$. From assertions 2 and 3 of Theorem 2 follows that the convergence rate of the mean square deviation of the sequential estimator $\vartheta_{\varepsilon}^{*}$ corresponds with the rate of convergence of the MLE in stationary and periodic cases [Gu/Ku].

According to the inequalities (29) the duration of observations $T(\varepsilon)$ in stationary case is approximately not great than $\varepsilon^{-1} \mu_{2}$ with $\mu_{2}$ defined by (30) when $\varepsilon$ is small. Note that in this case one can obtain the following limiting equalities

$$
\lim _{\varepsilon \rightarrow 0} \nu_{\varepsilon}=\nu \text { a.s. }
$$

and

$$
\lim _{\varepsilon \rightarrow 0} \varepsilon T(\varepsilon)=\mu_{2} \text { a.s. }
$$

Here $\nu$ is defined by (30). To obtain (32) we change the definition of $\nu_{\varepsilon}$ a little bit. Replace the magnitudes $\beta_{n}^{-2}(\varepsilon)$ in the definition of $\nu_{\varepsilon}$ in (11) by the nearest integer from above and choose $\left(c_{n}\right)$ in such a way that the constant $\varrho$ in (11) is irrational. In this case, the $\operatorname{limit}_{\varepsilon \rightarrow 0} \lim _{N}(\varepsilon)$ is stricly greate thn $\varrho$, and this implies (32).

From (32) it follows that by small $\varepsilon$ the moments $\nu_{\varepsilon}=\nu$ a.s. and by the property (28) it is obvious that the sequential estimate $\vartheta_{\varepsilon}^{*}$ may be represented in stationary case as the mean of finite numbers $\nu$ of maximum likelihood estimates $\hat{\vartheta}$ which are calculated at the moments $\tau_{\varepsilon}(n)$ :

$$
\vartheta_{\varepsilon}^{*} \sim \frac{1}{\nu} \sum_{n=1}^{\nu} \hat{\vartheta}\left(\tau_{\varepsilon}(n)\right)
$$

The number $\nu$ may be asymptotically estimated with help of the property (24) and by the definition (30) of the moment $\nu$.

It should be pointed out also that by known bound $\alpha$ for $\inf _{u \in[0, \Delta)} \beta^{2}(u) \geq \alpha>0$ with $\beta^{2}(u)$ defined by (15), according to (18) we obtain

$$
\nu_{\varepsilon} \leq \inf \left\{N \geq 1: N>\varrho \alpha^{-1}\right\}=1
$$

by small $\varepsilon$ if the sequence $\left(c_{n}\right)$ is such that $\varrho<\alpha$. Then for the sequential estimate $\vartheta_{\varepsilon}^{*}$ defined by (12) for small $\varepsilon$ we have

$$
\vartheta_{\varepsilon}^{*}=\hat{\vartheta}\left(\tau_{1}(\varepsilon)\right) \text { a.s. }
$$

Remark. From Theorem 2 we can see that the sequential estimators $\vartheta_{\varepsilon}^{*}$ converge to the true value $\vartheta$ in mean square as $\varepsilon \rightarrow 0$ in stationary and periodic cases. Moreover, for any sequence $\left(\varepsilon_{n}, n \geq 1\right)$ of positive integers such that $\sum_{n \geq 1} \varepsilon_{n}<\infty$, we can define the sequence of estimators $\left(\tilde{\vartheta}_{n}, n \geq 1\right), \tilde{\vartheta}_{n}=\vartheta_{\varepsilon_{n}}^{*}, n \geq 1$. Then the sequence $\left(\tilde{\vartheta}_{n}\right)$ of estimators for $\vartheta$ is strongly consistent. It follows from the assertion 2 of Theorem 2 and the Borel - Cantelli lemma. 


\section{References}

[Gu/Ku] Gushchin, A.A. and Küchler, U. Asymptotic Inference for a linear stochastic differential equation with time delay, to appear in Bernoulli.

[Ha/Ve] Hale, J.K. and Verduyn Lunel, S.M. (1993) Introduction to functionaldifferential equations, New York; Springer-Verlag.

[Ko/Pe] Konev, V.V. and Pergamenshchikov, S.M. (1985) Sequential estimation of the parameters of diffusion processes. Problems of Inform. Trans., 21, 1, 48-62.

[Ko/Pe] Konev, V.V. and Pergamenshchikov, S.M. (1987) Sequential estimation of the parameters of unstable dynamical systems in continuous time. Math. Stat. and Appl., Publishing House of Tomsk University, Tomsk, 11, 85-94.

[Ko/Pe] Konev, V.V. and Pergamenshchikov, S.M. (1992) Sequential estimation of the parameters of linear unstable stochastic systems with guaranteed accuracy. Problems of Inform. Trans., 28, 4, 35-48.

[Ku/Kut] Küchler and Kutoyants, Yu. A. (1998) Delay estimation for stationary diffustion-type process. Discussion Paper 47 of the SFB 373, HumboldtUniversity of Berlin, 1998.

[Ku/Me] Küchler, U. and Mensch, B. (1991) Langevins stochastic differential equations extended by a time-delayed term. Stochastics and Stochastic Reports, 40, $23-42$.

[Ku/So] Küchler, U. and Sorensen, M. (1997) Exponential Families of Stochastic Processes, New York, Heidelberg; Springer Verlag.

[Li/Sh] Liptzer, R.S. and Shiryaev A.N. (1977) Statistics of Random Processes, Vol 1,2. New York, Heidelberg; Springer Verlag.

[Mo] Mohammed, S.E-A. (1984) Stochastic Functional Differential Equations, Pitman, London.

[Mo/Sch] Mohammed, S.E-A. and Scheutzow, M.K.R. (1990) Lyapunov exponents and stationary solutions for affine stochastic delay equations. Stochastics and Stochastic Reports, 29, 259-283.

[Nov] Novikov, A.A. (1971) The sequential parameter estimation in the process of diffusion type. Probab. Theory and its Appl., 16, 2, 394-396.

[Va/Ko] Vasiliev, V.A. and Konev, V.V. (1987) On sequential identification of linear dynamic systems in continuous time by noisy observations. Probl. of Contr. and Inform. Theory, 16, 2, 101-112. 
[Va/Ko] Vasiliev, V.A. and Konev, V.V. (1990) On sequential parameter estimation of continuous dynamic systems by discrete time observations. Probl. of Contr. and Inform. Theory, 19, 3, 197-207. 